\title{
The Impact of Molecular Weight on the Behavior of Poly(propylene glycols) Derivatives Confined within Alumina Templates
}

Magdalena Tarnacka ${ }^{* *}$, Agnieszka Talik ${ }^{\uparrow}$, Ewa Kamińska ${ }^{\$}$, Monika Geppert-Rybczyńska ${ }^{\S}$, Kamil Kaminski ${ }^{\dagger *}$, Marian Paluch ${ }^{\ddagger}$

† Institute of Physics, University of Silesia, 75 Pulku Piechoty 1, 41-500 Chorzow, Poland

$\mp$ Silesian Center of Education and Interdisciplinary Research, University of Silesia, 75 Pulku Piechoty 1A, 41-500 Chorzow, Poland

\$ Department of Pharmacognosy and Phytochemistry, Medical University of Silesia in Katowice, School of Pharmacy with the Division of Laboratory Medicine in Sosnowiec, Jagiellonska 4, 41-200 Sosnowiec, Poland

§ Institute of Chemistry, University of Silesia, Szkolna 9, 40 -006 Katowice, Poland

* Corresponding authors: (MT) e-mail: magdalena.tarnacka@smcebi.edu.pl; $\quad$ (KK) email: kamil.kaminski@us.edu.pl; kamil.kamimski@smcebi.edu.pl 


\section{Experimental section.}

\section{Materials}

The Poly(propylene glycol) (PPG-OH) and Poly(propylene glycol) bis(2-aminopropyl ether) (PPG-NH ${ }_{2}$ ) samples with purity higher than $98 \%$ and molecular weights $\sim 400,2000,4000$ g/mol were supplied by Sigma-Aldrich. Poly(propylene glycol) dimethyl ether $\left(\mathrm{PPG}-\mathrm{OCH}_{3}\right)$ of various molecular weights were prepared accordingly to the synthesis reported in Ref. '(see description below). The applied anodic aluminum oxide (AAO) membranes used in this study (supplied from Synkera Co and InRedox) are composed of uniaxial channels (open from both sides) with well-defined pore diameter. The chemical structure of examined compounds is presented in Figure S1. Details concerning the porosity, pore diameter and distribution of AAO membranes are presented in Table S1.<smiles>[R]C1CCCC1CC(C)OCC(C)C(C)C1CCC1</smiles>

Poly(propylene glycol) derivatives where - $\mathrm{R}: \quad-\mathrm{OH} /-\mathrm{NH}_{2} /-\mathrm{OCH}_{3}$ (a) (b)

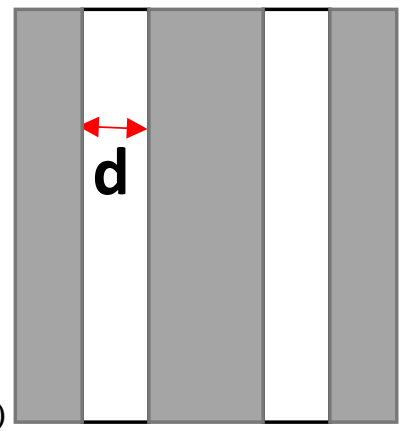

Fig. S1. The chemical structure of the investigated compounds (a) and the applied alumina constrain medium (b).

\begin{tabular}{|c|c|c|c|c|}
\hline Parameter & \multicolumn{4}{|c|}{ Membranes } \\
\hline Pore diameter $[\mathrm{nm}]$ & 18 & 35 & 73 & 150 \\
\hline Pore density $\left[\mathrm{cm}^{-2}\right]$ & $6 \cdot 10^{10}$ & $6 \cdot 10^{9}$ & $2 \cdot 10^{9}$ & $9 \cdot 10^{8}$ \\
\hline Pore period $[\mathrm{nm}]$ & 44 & 143 & 243 & 243 \\
\hline
\end{tabular}

Table S1. Details concerning porosity, pore diameter and distribution of AAO membranes. 


\section{Methods.}

\subsection{Synthesis of PPG-OCH3}<smiles>COC(C)COCC(C)OCC(C)OCC(C)OCC(C)OCC(C)OCC(C)O</smiles>

The mixture of $10 \mathrm{~g}$ ( $2.5 \mathrm{mmol})$ of hydroxyl-functionalized PPG and $0.24 \mathrm{~g}(6 \mathrm{mmol} ; 2.4 \mathrm{eq})$ of $\mathrm{NaH}(60 \%$ in mineral oil) was stirred under inert gas atmosphere for $24 \mathrm{~h}$ in a two-neck bottom flask equipped with magnetic stirrer and rubber septum. Then $\mathrm{CH}_{3} \mathrm{I}$ was added dropwise and stirring was continued for additional $24 \mathrm{~h}$. After that the reaction was terminated by adding $40 \mathrm{ml}$ of methanol. The post-reaction mixture was purified by ultrafiltration followed by evaporation of the volatiles fractions. The residue was dissolved in dichloromethane and the organic solution was washed with water (three times) and next dried over $\mathrm{MgSO}_{4}$. PPG-OCH 3 was received as a pale-yellow oil with $92 \%$ yield. The product of the reaction was confirmed by IR spectra, see Figure S2.

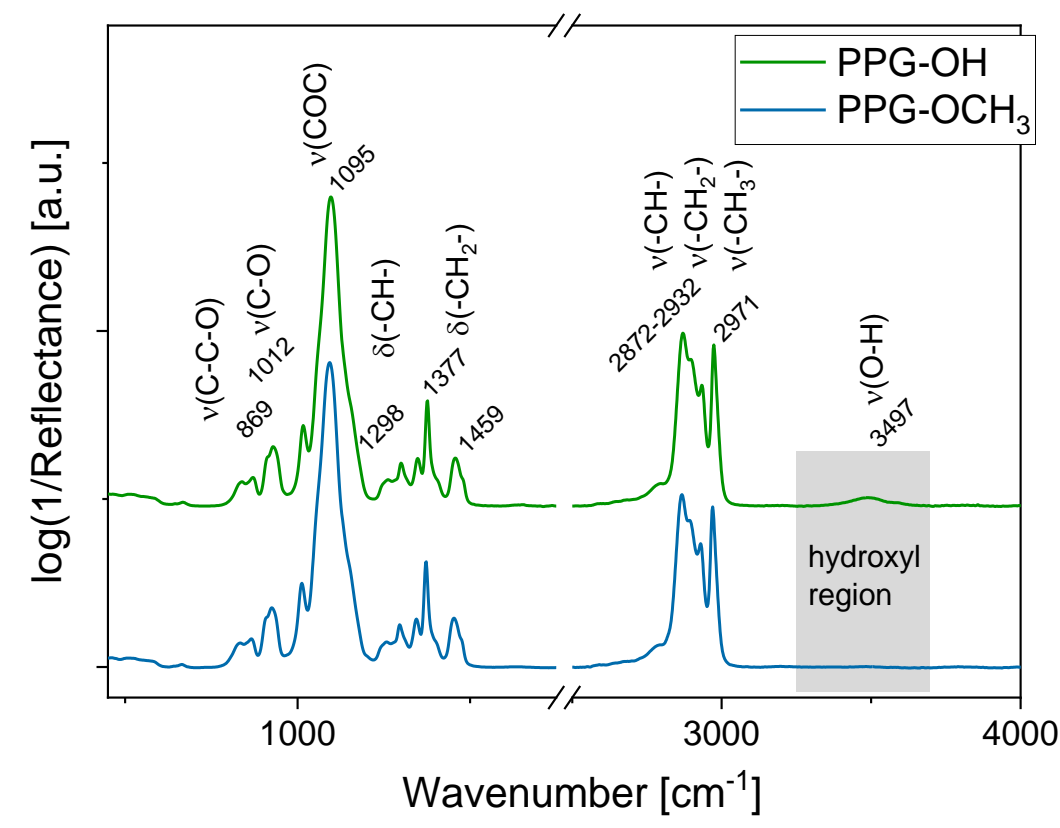

Fig. S2. IR spectra collected before and after the synthesis of PPG-OCH$H_{3}$. Data taken from Ref. 1. 


\subsection{Surface Tension and Contact Angle Measurements.}

The solid surface energy of aluminum oxide, $\gamma_{S}$, was calculated using the surface tension and contact angles for test liquids (water and ethylene glycol) according to Fowkes method. ${ }^{2,3}$ This procedure regards the surface tension, $\gamma_{L}$, as a sum of dispersive, $d$, and non-dispersive components, $n d$ : $\left(\gamma_{L}=\gamma_{L}^{d}+\gamma_{L}^{n d}\right)$. The non-dispersive contribution is the sum of all parts of $\gamma_{L}$ resulting from non-dispersion intermolecular interactions present in a liquid (the same is for solid) such as hydrogen bonds and base-acid interactions. Since the contact angle, $\theta$, is related to surface energy, $\gamma_{S}$, interfacial energy, $\gamma_{S L}$, and to the surface tension, $\gamma_{L}$, accordingly to the Young equation is:

$$
\gamma_{S L}=\gamma_{S}-\gamma_{L} \cos \theta
$$

The work of adhesion is equal:

$$
W_{a}=\gamma_{L}+\gamma_{S}-\gamma_{S L}
$$

and assuming that:

$$
W_{a}=2\left(\sqrt{\left(\gamma_{S}^{d} \cdot \gamma_{L}^{d}\right)}+\sqrt{\left(\gamma_{S}^{n d} \cdot \gamma_{L}^{n d}\right)}\right)
$$

the solid surface energy can be calculated with dispersive and non-dispersive components accordingly to the following relation: ${ }^{4}$

$$
\frac{\gamma_{L \cdot(1+\cos \theta)}}{2 \cdot \sqrt{\gamma_{L}^{d}}}=\sqrt{\gamma_{S}^{n d}} \sqrt{\frac{\gamma_{L}^{n d}}{\gamma_{L}^{d}}}+\sqrt{\gamma_{S}^{d}},
$$

The Fawkes approach gives a reliable estimation of the surface energy, what was reported earlier. ${ }^{5,6,7}$ Previously estimated solid surface energy of aluminum oxide was equal to $\gamma_{S}=$ $58.97 \cdot 10^{-3} \mathrm{~N} \cdot \mathrm{m}^{-1}$, with the non-dispersive part $\gamma_{S}^{\text {nd }}=55.6 \cdot 10^{-3} \mathrm{~N} \cdot \mathrm{m}^{-1} \cdot{ }^{1}$ Based on $\gamma_{S}$, the surface tension and the contact angle of various compounds on AAO, the interfacial energy at 
$\mathrm{T}=298 \mathrm{~K}$ was calculated from the Eq. (S1) and listed in Table S2, as well as plotted versus $\Delta \mathrm{T}_{\mathrm{g}}$ and $\Delta \mathrm{T}_{\mathrm{c}}$ in Figure 7.

\begin{tabular}{|c|c|c|c|}
\hline Sample & $\theta\left[{ }^{0}\right]$ & $\gamma_{L}\left[\mathbf{m N ~ m}^{-1}\right]$ & $\begin{array}{c}\gamma_{\mathrm{SL}} \text { at } \mathrm{T}=298 \mathrm{~K} \\
{\left[\mathrm{mN} \mathrm{m^{-1 } ]}\right.}\end{array}$ \\
\hline \multicolumn{4}{|c|}{$\mathrm{M}_{\mathrm{n}}=4000 \mathrm{~g} / \mathrm{mol}$} \\
\hline PPG-OH & 8.35 & 32.2 & 27.2 \\
\hline PPG-NH 2 & 10 & 31.9 & 27.5 \\
\hline PPG-OCH 3 & 14.0 & 31.6 & 27.9 \\
\hline \multicolumn{4}{|c|}{$\mathrm{M}_{\mathrm{n}}=2000 \mathrm{~g} / \mathrm{mol}$} \\
\hline PPG-OH & 7.2 & 32.0 & 27.2 \\
\hline PPG-NH 2 & 7.1 & 31.4 & 27.9 \\
\hline \multicolumn{4}{|c|}{$\mathrm{M}_{\mathrm{n}}=400 \mathrm{~g} / \mathrm{mol}$} \\
\hline PPG-OH & 6.2 & 32.4 & 26.7 \\
\hline PPG-NH 2 & 5.4 & 31.6 & 27.6 \\
\hline PPG-OCH ${ }_{3}$ & 6 & 22.8 & 36.3 \\
\hline
\end{tabular}

Table S2. Contact angle, surface tension and solid - liquid interfacial tension at $\mathrm{T}=298 \mathrm{~K}$ for investigated samples. Data for PPG derivatives of $M_{n}=4000 \mathrm{~g} / \mathrm{mol}$ were taken from Ref. 1 .

This procedure was applied using at first the software recommended by the provider for the Kruess tensiometer, GmbH, Germany included in DSA 100S software, and then it was repeated independently taking into account: water, ethylene glycol, diiodomethane and bromobenzene. Finally, only two from these liquids were taken into account: water and ethylene glycol (with a stable and repeatable contact angle values on AAO), since for diiodomethane and bromobenzene contact angle on alumina was around $0^{\circ}$. We did not regard glycerol due to similar ratio of dispersive and non-dispersive part in the surface tension to ethylene glycol. We believe that taking more liquids for calibration can cause also some calculations problems as was referred by Jańczuk et al. ${ }^{3}$ and Hejda et al. ${ }^{5}$. Moreover, it should be mentioned that for all samples (there were all in a liquid state) the difference between the advancing and receding contact angles was below the estimated uncertainty of the contact angle measurements; thus, for all calculations the mean value was taken into account. Therefore in contrast to the other polymers of larger molecular weight in PPG the advancing and receding contact angles seems to be comparable. 


\subsection{Differential Scanning Calorimetry (DSC).}

Calorimetric measurements were carried out using a Mettler-Toledo DSC apparatus (MettlerToledo International, Inc., Greifensee, Switzerland) equipped with a liquid nitrogen cooling accessory and an HSS8 ceramic sensor (heat flux sensor with 120 thermocouples). Temperature and enthalpy calibrations were investigated using indium and zinc standards, while the heat capacity, $\mathrm{C}_{\mathrm{p}}$, calibration was performed using a sapphire disc. Measurements were carried out on bulk and the confined PPG derivatives of different molecular mass. Bulk samples were contained in sealed alumina crucibles, and the heating rate was equal to $10 \mathrm{~K} / \mathrm{min}$ over a temperature range from $170 \mathrm{~K}$ to $300 \mathrm{~K}$, see Fig. S3. In the case of confined systems, the filled membranes were crushed to fix the measurement alumina crucibles. The samples were contained in sealed crucibles with the standard heating rate of $10 \mathrm{~K} / \mathrm{min}$, see Fig. S3. Additionally, PPG-OH of $\mathrm{M}_{\mathrm{n}}=400 \mathrm{~g} / \mathrm{mol}$ and $\mathrm{M}_{\mathrm{n}}=4000 \mathrm{~g} / \mathrm{mol}$ infiltrated into AAO templates of $d=18 \mathrm{~nm}$ have been measured with additional heating rates ( 5 and $20 \mathrm{~K} / \mathrm{min}$ ). Representative DSC thermograms obtained for confined PPG-OH of $M_{n}=400 \mathrm{~g} / \mathrm{mol}$ are presented in Fig. S4. 

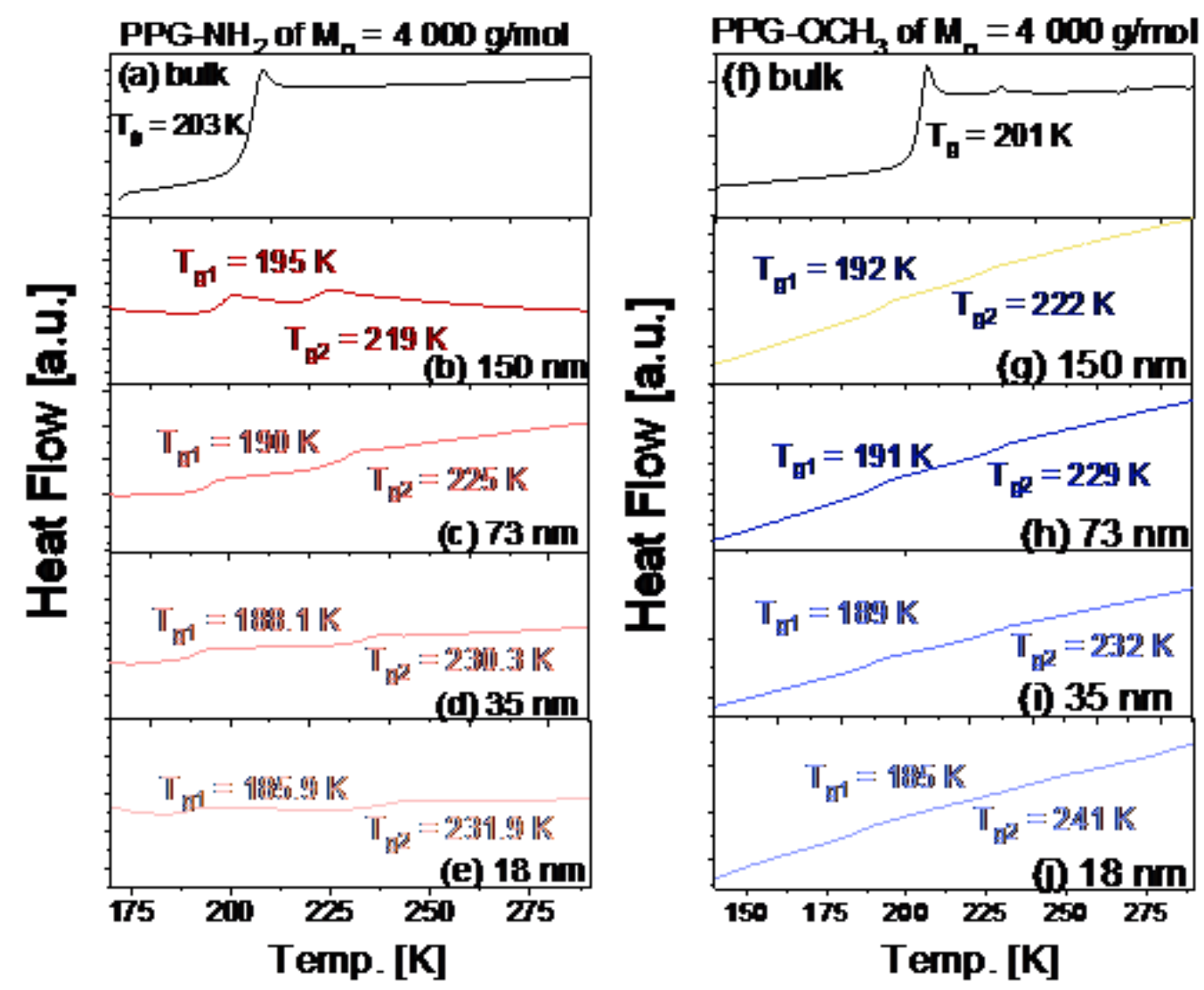

Fig. S3. DSC thermograms of bulk and confined PPG-NH $2(a-e)$ and PPG-OCH $(f-j)$. Note that bulk data were added for better data presentation and to highlight that confined $\mathrm{T}_{g} \mathrm{~S}$ are located below and above bulk $\mathrm{T}_{g}$.

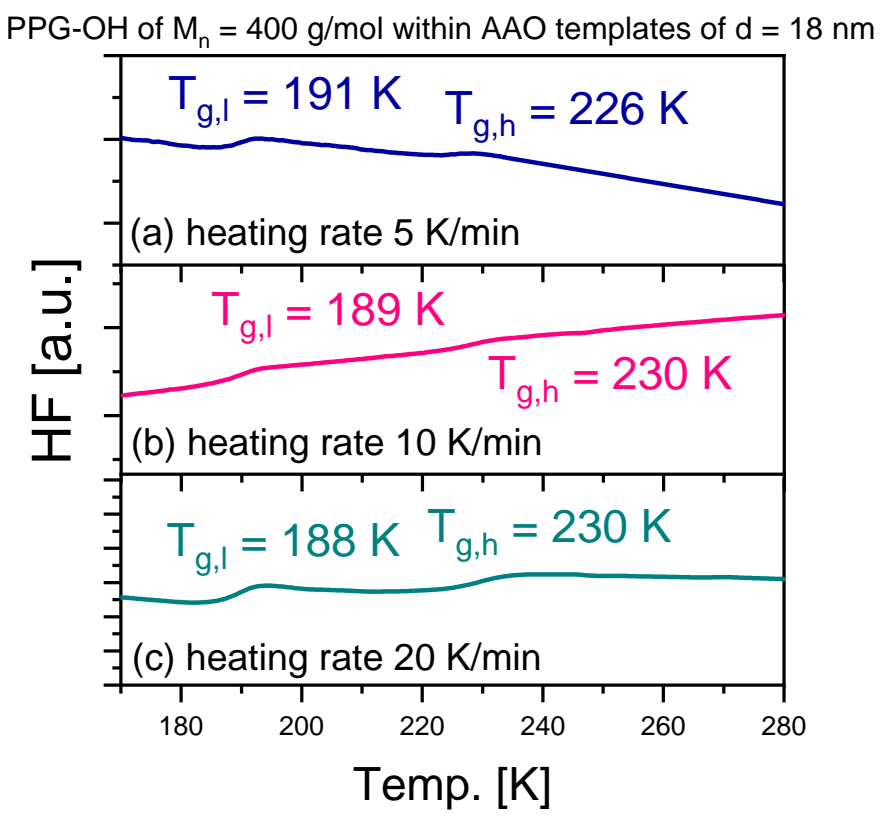

Fig. S4. DSC thermograms collected for PPG-OH of $\mathrm{M}_{\mathrm{n}}=400 \mathrm{~g} / \mathrm{mol}$ infiltrated into AAO templates of $d=18 \mathrm{~nm}$ measured at different heating rates: 5 (a), 10 (b) and $20 \mathrm{~K} / \mathrm{min}$ (c). 
The comparison of dielectric spectra collected for bulk and confined PPG derivatives of various $\mathbf{M}_{\mathbf{n}}$
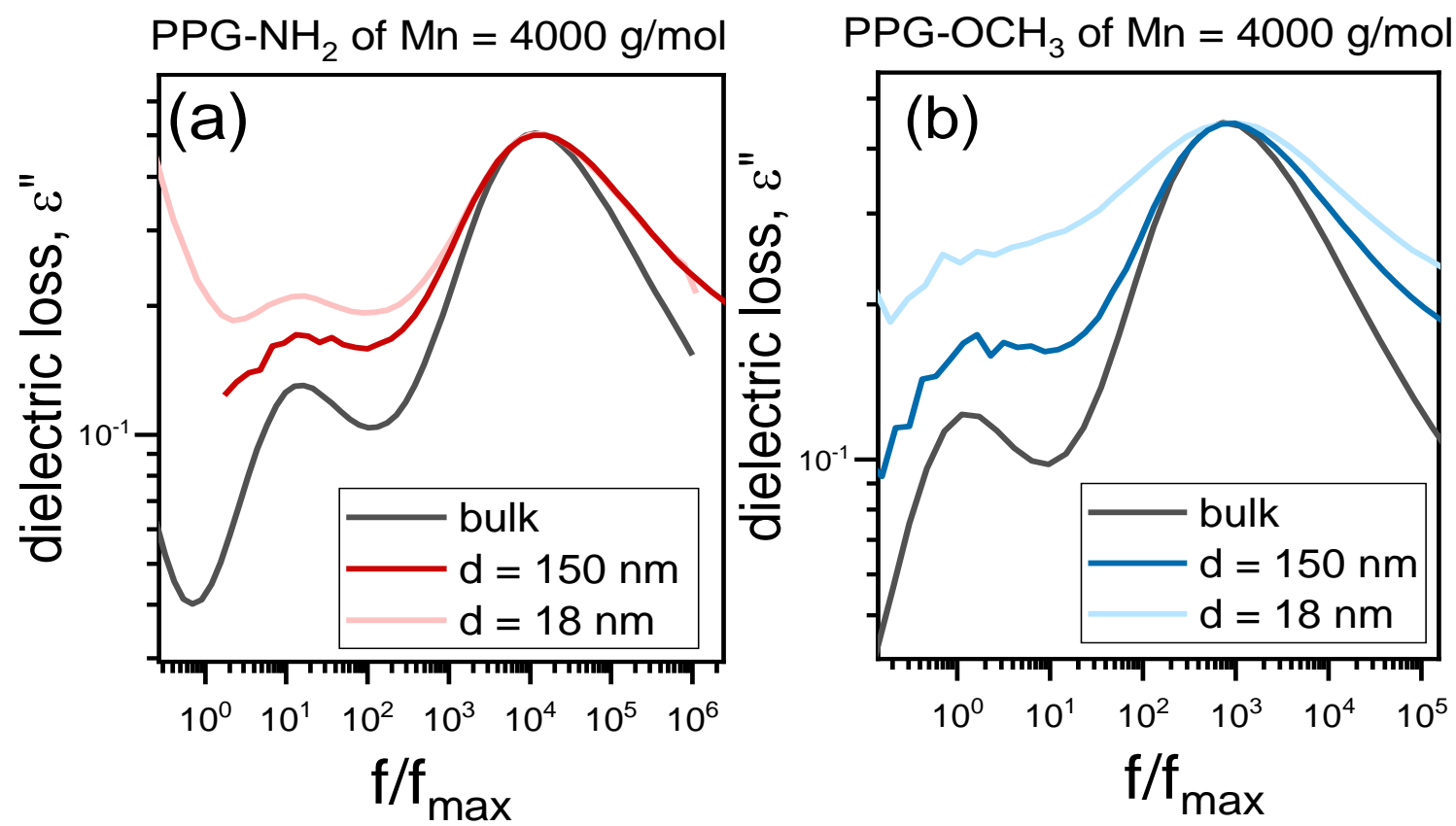

Fig. S5. Comparison of the segmental relaxation shape recorded for the bulk and confined PPG-NH $\mathrm{N}_{2}$ (a) and PPG-OCH $\mathrm{P}_{3}$ (b) incorporated in two pore sizes $d=18 \mathrm{~nm}$ and $d=150 \mathrm{~nm}$.

(a) $\mathrm{d}=18 \mathrm{~nm}$

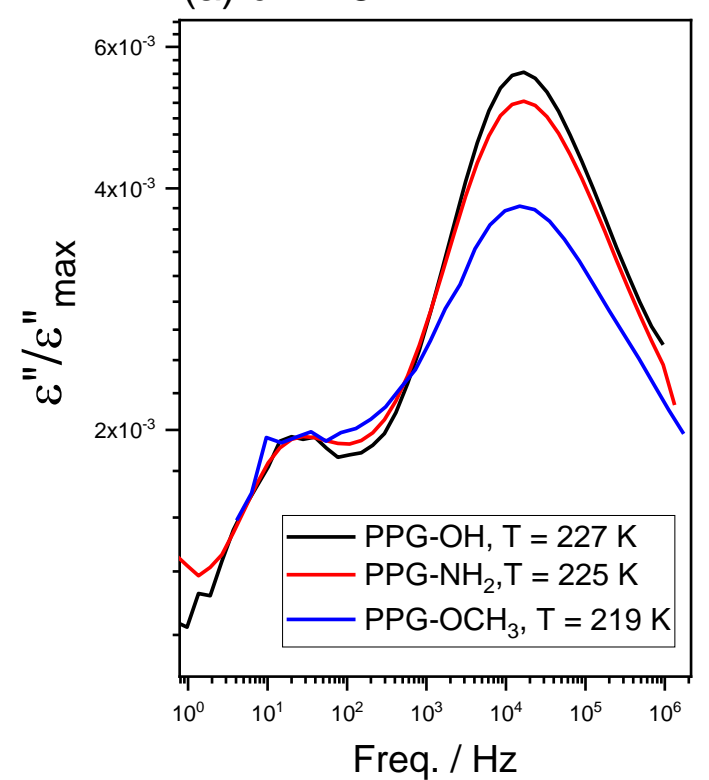

(b) $\mathrm{d}=150 \mathrm{~nm}$

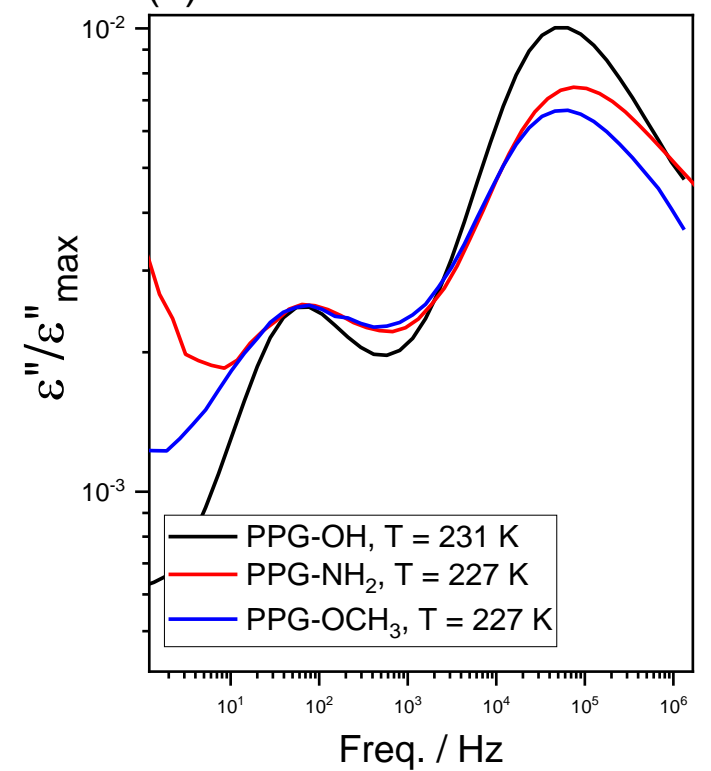

Fig. S6. Comparison of the shape of NM process of the studied PPG derivatives of $M_{n}=4$ $000 \mathrm{~g} / \mathrm{mol}$ confined within AAO templates of different pore size $d=18 \mathrm{~nm}$ (a) and $d=150$ $\mathrm{nm}(\mathrm{b})$. 


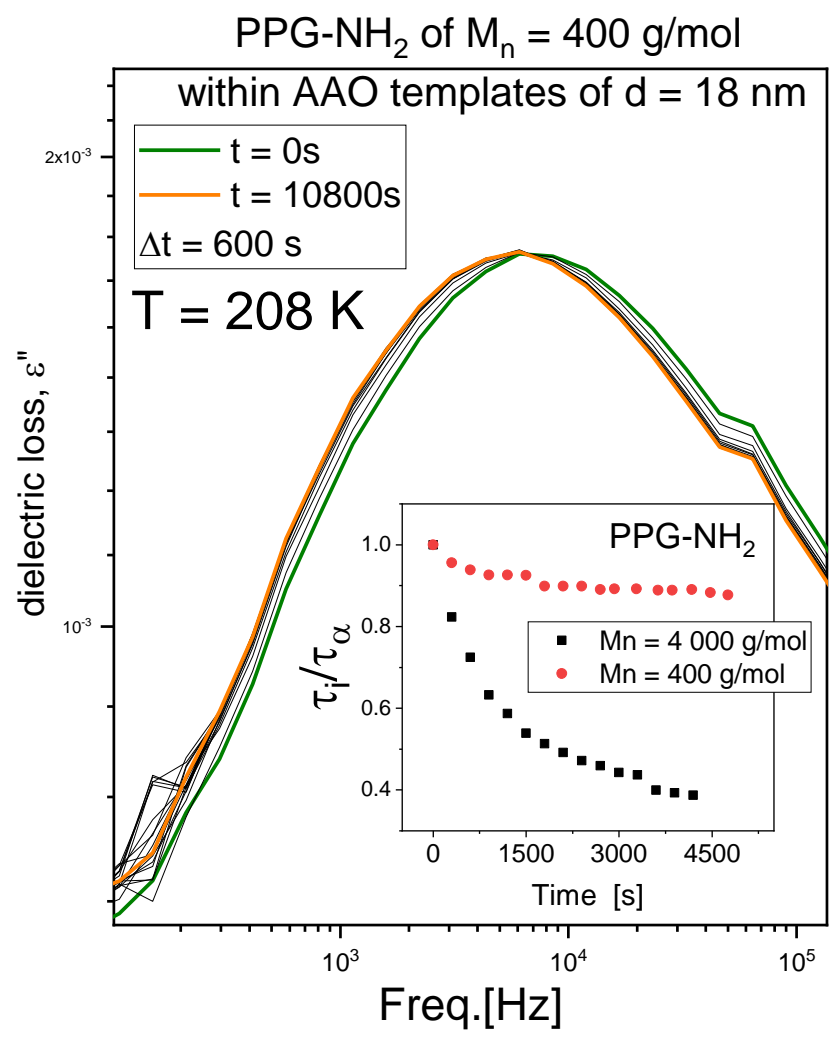

Fig. S7. Time evolution of the loss spectra measured for PPG-NH $\mathrm{N}_{2}$ of $\mathrm{M}_{\mathrm{n}}=400 \mathrm{~g} / \mathrm{mol}$ confined in AAO templates of $d=18 \mathrm{~nm}$ at $\mathrm{T}_{\text {anneal }}=208 \mathrm{~K}$; As the inset, time dependence of $\tau_{\mathrm{i}} / \tau_{\alpha}$ for PPG-NH${ }_{2}$ of $\mathrm{M}_{\mathrm{n}}=400 \mathrm{~g} / \mathrm{mol}$ and $\mathrm{M}_{\mathrm{n}}=4000 \mathrm{~g} / \mathrm{mol}$ confined within AAO templates of $d=18 \mathrm{~nm}$.

\section{References}

(1) Talik, A.; Tarnacka, M.; Grudzka-Flak, I.; Maksym, P.; Geppert-Rybczyńska, M.; Kaminska, E.; Kaminski, K.; Paluch, M. The Role of Interfacial Energy and Specific Interactions on the Behavior of Poly(propylene Glycols) Derivatives under 2D Confinement Macromolecules 2018, 51, 4840-4852.

(2) Fowkes, F. M. Attractive Forces and Interfaces. Ind. Eng. Chem. 1964, 56, 40-52.

(3) Jańczuk, B.; Białopiotrowicz, T.; Zdziennicka, A.; Some Remarks on the Components of the Liquid Surface Free Energy. J. Coll. Interface Sci. 1999, 211, 96-103.

(4) Vicente, C. M. S.; André, P. S.; Ferreira, R. A. S. Simple Measurement of Surface Free Energy using a Web Cam. Rev. Bras. Ensino Fis. 2012, 34, 3312.

(5) Hejda, F.; Solař, P.; Kousal, J. Surface Free Energy Determination by Contact Angle Measurements - A Comparison of Various Approaches. WDS'10 Proceedings of Contributed Papers, Part III. 2010, 25-30. 
(6) Kalin, M.; Polajnar, M. The Correlation between the Surface Energy, the Contact Angle and the Spreading Parameter, and their Relevance for the Wetting Behavior of DLC with Lubricating Oils. Tribol. Int. 2013, 66, 225-233.

(7) Kalin, M.; Polajnar, M. The Wetting of Steel, DLC Coatings, Ceramics and Polymers with Oils and Water: The Importance and Correlations of Surface Energy, Surface Tension, Contact Angle and Spreading. Appl. Surf. Sci. 2014, 293, 97-108. 